\title{
Consistance des systèmes de Lur'e commutés Application à la synthèse de commande numérique avec un échantillonnage non uniforme
}

\author{
Julien Louis $^{1,2}$, Marc Jungers ${ }^{1,2}$, Jamal Daafouz ${ }^{1,2,3}$ \\ 1. Centre de Recherche en Automatique de Nancy, \\ UMR 7039 - Université de Lorraine, CNRS \\ 2, Avenue de la forêt de Haye. 54516 Vandouvre, France \\ [julien.louis;marc.jungers;jamal.daafouz]@univ-lorraine.fr \\ 2. CNRS, CRAN, UMR 7039, France \\ 3. Institut Universitaire de France
}

\begin{abstract}
RÉSUMÉ. Dans cet article, à partir d'une relecture de la consistance des systèmes commutés linéaires, la propriété de consistance pour les systèmes non-linéaires de type Lur'e commutés à temps discret est étudiée. Une discussion montre qu'il faut considérer le plus petit majorant quadratique possible de la performance au lieu de la valeur optimale elle-même (qui coüncident dans le cadre linéaire) pour permettre l'extension. Un problème d'optimisation sous contraintes LMI est proposé pour obtenir une stratégie de type min-switching satisfaisant la consistance en non-linéaire. Dans un second temps, cette contribution est utilisée pour la synthèse d'un contrôleur à données échantillonnées avec un échantillonnage non uniforme. Un exemple numérique est alors proposé pour mettre en évidence les apports de ces résultats.

ABSTRACT. In this paper, starting from a rereading of the consistency notion for switched linear systems, the consistency is studied for discrete-time switched Lur'e type systems. A discussion highlights that the smallest upper bound of the cost function should be considered instead of the optimal value (even if they coincide in the linear case) to allow the extension in the nonlinear framework. An optimization problem with LMI constraints is provided to design a minswitching strategy that is consistent in the nonlinear case. Moreover this contribution is used for the synthesis of a sampled data controller with non uniform sampling. A numerical illustration is proposed to show the relevance of these results.

MOTS-CLÉS : Système non linéaire de type Lur'e, commande à données échantillonnées, échantillonnage non uniforme, consistance des systèmes commutés.

KEYWORDS: LUr'e type nonlinear systems, sampled data control, non-uniform sampling, consistency of switched systems.
\end{abstract}




\section{Introduction}

Les systèmes commutés représentent une classe de systèmes hybrides avec des caractéristiques spécifiques qui les rendent particulièrement intéressants. En particulier, une loi de commutation peut être capable de stabiliser une famille de modes instables ou bien au contraire, de rendre instable une famille de modes stables (Liberzon, 2003). Plus récemment, une autre propriété intéressante a été mise en évidence (Geromel $e t$ al., 2011) : sous certaines conditions, la synthèse de la loi de commutation améliore la performance du système commuté linéaire par rapport à la performance obtenue en considérant un sous-système isolé. Ainsi, dans le cas où cette propriété de consistance est vérifiée, la loi de commutation ne peut pas dégrader le niveau de performance par rapport aux niveaux obtenus sans commuter. Plus précisément, cette propriété a été étudiée dans le cadre des systèmes commutés linéaires associés aux fonctions coût de type $\mathscr{H}_{2}$ et $\mathscr{H}_{\infty}$ (Geromel et al., 2011; Souza et al., 2012; Deaecto, Souza, Geromel, 2013; Deaecto, Fioravanti, Geromel, 2013; Geromel et al., 2013).

Les systèmes non linéaires composés de l'interconnexion entre un système linéaire et une non-linéarité vérifiant une condition de secteur de type cône borné sont nommés systèmes de Lur'e. Cette classe de systèmes a été largement étudiée par la communauté scientifique. Son introduction dans l'article (Lur'e, Postnikov, 1944) a permis l'étude de la stabilité absolue, c'est-à-dire la stabilité de cette interconnexion pour toute non-linéarité vérifiant une condition de secteur de type cône borné connue. La littérature offre plusieurs extensions de ce cadre, notamment pour de tels systèmes commutés : en temps continu (Castelan et al., 2008) ou bien en temps discret (Jungers et al., 2011). Les résultats associés considèrent des fonctions de Lyapunov quadratiques commutées, mais aussi des fonctions de Lyapunov plus adaptées (Gonzaga et al., 2012b ; 2012a ; Hajiahmadi et al., 2014). Au-delà de la stabilité, en associant un critère de performance à ce système, le problème se complique. A cause de la nature non linéaire du problème, il est difficile d'obtenir simplement des solutions optimales pour un certain critère de performance. Dans le cas linéaire, les méthodes de Lyapunov permettent de déterminer la valeur exacte du coût. Ces outils, relatifs au cas linéaire, ne s'appliquent plus dans le cadre non linéaire. Ils restent néanmoins utiles pour obtenir des majorants quadratiques des coûts. Cette observation met en exergue le fait que la propriété de consistance (Geromel et al., 2011) peut être étudiée en ce qui concerne les majorants quadratiques des coûts.

Cet article a deux objectifs. A partir d'une relecture de la notion de consistance pour les systèmes commutés linéaires à temps discret, le premier but est de proposer pour la première fois des conditions suffisantes de consistance pour les systèmes commutés non-linéaires de type Lur'e. Ainsi nous proposerons une synthèse de loi de commutation fondée sur la stratégie de min-switching garantissant que les majorants quadratiques des coûts vérifient le principe de consistance. Le second objectif est d'utiliser cette approche pour implémenter un contrôleur à données échantillonnées avec un échantillonnage non uniforme. Les systèmes à données échantillonnées ont été largement traités. Il est possible de distinguer trois grandes familles de synthèse de contrôleur (Monaco, Normand-Cyrot, 2001) : 
- la synthèse à temps continu (STC), qui suggère d'implémenter directement une version discrétisée du contrôleur à temps continu (Monaco, Normand-Cyrot, 1985; Hsu, Sastry, 1987);

- la synthèse à temps discret (STD), qui propose de stabiliser le système uniquement sur les échantillons (Barbot et al., 1996);

- la synthèse à données échantillonnées (SDE), qui complète l'approche STD en tenant compte du comportement des trajectoires entre deux échantillons consécutifs (Nešić, Teel, Sontag, 1999; Nešić, Teel, Kokotović, 1999; Laila et al., 2005).

Dans cet article, nous nous attachons à l'étude d'une solution issue de la méthode STC avec un échantillonnage non uniforme. L'idée est d'attribuer une fonction coût à chaque période d'échantillonnage et de proposer une stratégie menant à un échantillonnage non uniforme minimisant un majorant de cette fonction coût et qui puisse augmenter la période moyenne d'échantillonnage.

L'article est organisé comme suit. La section 2 permet de rappeler la notion de consistance pour les systèmes commutés linéaires à temps discret et de mettre en avant les difficultés afférentes à l'extension au cas non-linéaire. La section 3 traite de la propriété de consistance pour les systèmes de Lur'e commutés à temps discret. La section 4 offre une application à la synthèse de contrôleur à données échantillonnées. Un exemple est donné en considérant une méthode STC avec deux périodes d'échantillonnage possibles. Une conclusion est proposée à la section 5 .

Notation. Pour tout vecteur $x \in \mathbb{R}^{n}, x \geq 0$ signifie que pour $\forall i=\{1 \cdots n\}, n \in$ $\mathbb{N}$, chaque composante $x_{(i)}$ est positive ou nulle. Pour deux matrices $A$ et $B \in \mathbb{R}^{n \times n}$, $A>B$ signifie que la matrice $A-B$ est définie positive. $A^{\prime}$ représente la transposée de la matrice $A$. $I_{n}$ est la matrice identité d'ordre $n$ et $0_{n \times p}$ la matrice nulle d'ordre $n \times p . \mathbf{1}_{n}$ est le vecteur colonne de dimension $n \times 1$ dont chaque élément est égal à 1 . Le symbole $\star$ représente un bloc symétrique dans les matrices. Nous introduisons $\mathscr{I}_{N}=\{1 ; \cdots ; N\}$, où $N$ est un entier positif.

\section{Consistance pour les systèmes commutés linéaires à temps discret}

Cette section est dédiée aux rappels des résultats de consistance pour les systèmes linéaires à temps discret développés dans (Geromel et al., 2011 ; Deaecto et al., 2011 ; Deaecto, Fioravanti, Geromel, 2013). Nous avons opté pour l'option qui consiste à présenter ces résultats sous une forme unifiée pour les comparer dans la section suivante avec les résultats que nous proposons dans le cadre des systèmes de type Lur'e (voir (Louis, 2015) pour plus de détails).

Nous choisissons d'illustrer la notion de consistance dans le cadre linéaire avec la classe suivante de systèmes linéaires commutés à temps discret :

$$
\left\{\begin{aligned}
x_{k+1} & =A_{\sigma(k)} x_{k}+H_{\sigma(k)} w_{k}, \\
z_{k} & =E_{\sigma(k)} x_{k}+G_{\sigma(k)} w_{k},
\end{aligned}\right.
$$


évoluant à partir de la condition initiale $x_{0}$, où $x_{k} \in \mathbb{R}^{n}$ est le vecteur d'état, $z_{k} \in \mathbb{R}^{p}$ est le vecteur de sortie du système et $w_{k} \in \mathbb{R}^{r}$ est une entrée exogène (perturbation ou commande). La loi de commutation $\sigma: \mathbb{N} \rightarrow \mathscr{I}_{N}$ est sélectionnée à chaque instant de temps $k \in \mathbb{N}$ parmi les $N$ sous systèmes considérés.

Un critère quadratique est associé à ce système (1) sous la forme :

$$
\mathscr{J}_{\sigma}\left(x_{0} ;\left\{w_{k}\right\}_{k \in \mathbb{N}}\right)=\sum_{k \in \mathbb{N}}\left(\begin{array}{c}
x_{k} \\
w_{k}
\end{array}\right)^{\prime} Q_{\sigma(k)}\left(\begin{array}{c}
x_{k} \\
w_{k}
\end{array}\right),
$$

avec $Q_{i}$ des matrices symétriques définies positives de dimensions appropriées. Il faut noter que la structure générique de ce coût $\mathscr{J}_{\sigma}(\cdot ; \cdot)$ permet d'englober l'étude de différents critères. Effectivement, nous pouvons considérer :

- le gain " $\mathscr{H}_{2}$ ", en posant :

$$
\mathscr{J}_{\sigma}\left(x_{0} ; 0\right)=\sum_{k \in \mathbb{N}}\left\|z_{k}\right\|^{2}
$$

$\operatorname{avec} \forall i \in \mathscr{I}_{N}$,

$$
Q_{i}=\left[\begin{array}{cc}
E_{i}^{\prime} E_{i} & \star \\
0_{r \times n} & 0_{r \times r}
\end{array}\right]
$$

- le coût induit $\mathscr{L}_{2}$, en posant :

$$
\mathscr{J}_{\sigma}\left(0 ;\left\{w_{k}\right\}_{k \in \mathbb{N}}\right)=\sum_{k \in \mathbb{N}}\left\|z_{k}\right\|^{2}-\rho\left\|w_{k}\right\|^{2}
$$

soit $\forall i \in \mathscr{I}_{N}$,

$$
Q_{i}=\left[\begin{array}{cc}
E_{i}^{\prime} E_{i} & \star \\
G_{i}^{\prime} E_{i} & G_{i}^{\prime} G_{i}-\rho I_{r}
\end{array}\right],
$$

et où le gain induit $\mathscr{L}_{2}$, noté $\gamma$, est défini tel que

$$
\gamma^{2}=\sup _{w \in \mathscr{L}_{2} \backslash\{0\}} \frac{\|z\|^{2}}{\|w\|^{2}},
$$

qui est alors majoré par :

$$
\gamma \leq \sqrt{\rho}
$$

Avant d'introduire la notion de consistance, nous définissons pour plus de clarté l'ensemble $\mathscr{S}$ contenant toutes les lois de commutations, prenant la forme d'un retour d'état c'est-à-dire dont $\sigma(k)$ est une fonction de $x_{k}$, qui assurent la stabilité asymptotique globale du système (1). Nous définissons également l'ensemble $\mathscr{C}$ contenant les $N$ lois de commutation maintenues constantes, c'est-à-dire telles que $\forall k \in \mathbb{N}$, $\sigma(k)=i \in \mathscr{I}_{N}$. Le concept de consistance est défini comme suit :

DÉFInition 1. - (Geromel et al., 2011). Considérons la classe des systèmes commutés linéaires à temps discret (1), une stratégie de commutation particulière $\sigma_{s}(\cdot)$ 
est dite consistante par rapport au critère $\mathscr{J}_{\sigma}(\cdot ; \cdot)$ défini par (2), si elle appartient à l'ensemble $\mathscr{S}$ et si elle garantit $\forall \sigma \in \mathscr{C}$, et $\forall x_{0} \in \mathbb{R}^{n}$ que

$$
\mathscr{J}_{\sigma_{s}}\left(x_{0} ;\left\{w_{k}\right\}_{k \in \mathbb{N}}\right) \leq \mathscr{J}_{\sigma}\left(x_{0} ;\left\{w_{k}\right\}_{k \in \mathbb{N}}\right) .
$$

Si l'inégalité est stricte, la stratégie de commutation est dite strictement consistante.

Une stratégie de commutation est dite consistante, si la performance associée est inférieure ou égale à la meilleure performance obtenue par un des sous systèmes étudiés indépendamment des autres. Une stratégie de commutation de type min-switching semble naturellement candidate pour garantir une telle propriété. Cependant la construction d'une telle loi de commutation dépend de la performance étudiée.

Le problème qui nous intéresse est alors décrit de la manière suivante :

Problème 2. - Construire une loi de commutation $\sigma_{s}$ telle que la propriété de consistance de la définition 1 soit satisfaite dans le cadre de la fonction coût (3) (respectivement (5)).

La stratégie de type min-switching se construit en considérant les outils suivants :

- la fonction de Lyapunov,

$$
V_{\min }:\left\{\begin{aligned}
\mathbb{R}^{n} & \rightarrow \mathbb{R}^{+}, \\
x_{k} & \mapsto \min _{i \in \mathscr{I}_{N}} V_{i}\left(x_{k}\right),
\end{aligned}\right.
$$

avec $\forall i \in \mathscr{I}_{N}, V_{i}\left(x_{k}\right)=x_{k}^{\prime} P_{i} x_{k}$ où $P_{i} \in \mathbb{R}^{n \times n}$ sont des matrices symétriques définies positives.

- l'ensemble des matrices de Metzler,

$$
\mathscr{M}=\left\{\Pi \in \mathbb{R}^{N \times N}, \forall(i, \ell) \in \mathscr{I}_{N}^{2}, \pi_{\ell i} \in[0,1], \pi_{\ell i} \geq 0, \sum_{\ell \in \mathscr{I}_{N}} \pi_{\ell i}=1\right\} .
$$

- la combinaison linéaire des matrices définissant la fonction de Lyapunov (10) fonction implicite des paramètres de la matrice de Metzler (11).

$$
(P)_{p, i}=\sum_{i \in \mathscr{I}_{N}} \pi_{j i} P_{j}
$$

Le théorème suivant résout le problème 2 dans le cadre de la performance (3).

THÉORÈME 3. - Soit le système (1) tel que l'entrée de perturbation est nulle. S'il existe des matrices symétriques définies positives $P_{i}, \forall i \in \mathscr{I}_{N}$, et une matrice de Metzler $\Pi \in \mathscr{M}$ solution du problème d'optimisation

$$
\min _{P_{i}, \Pi}\left(\min _{i \in \mathscr{I}_{N}} \operatorname{trace}\left(P_{i}\right)\right),
$$

sous les contraintes

$$
\left[\begin{array}{ccc}
P_{i} & \star & \star \\
(P)_{p, i} A_{i} & (P)_{p, i} & \star \\
E_{i} & 0 & I_{p}
\end{array}\right]>0, \quad \forall i \in \mathscr{I}_{N}
$$


alors la loi de commutation définie par $\sigma_{s}\left(x_{k}\right)=\arg \min _{i \in \mathscr{I}_{N}} x_{k}^{\prime} P_{i} x_{k}$ stabilise globalement, uniformément et asymptotiquement le système (1), et $\mathscr{J}_{\sigma_{s}}\left(x_{0} ;\left\{w_{k}\right\}_{k \in \mathbb{N}}\right) \leq V_{\min }\left(x_{0}\right)$. De plus cette loi de commutation est consistante.

PREUVE. - D'après (Geromel et al., 2008, théorème 1), si les contraintes (14) sont vérifiées, alors le système (1) est globalement, uniformément et asymptotiquement stable et que $\mathscr{J}_{\sigma_{s}}\left(x_{0} ;\left\{w_{k}\right\}_{k \in \mathbb{N}}\right) \leq V_{\min }\left(x_{0}\right)$. La stratégie de min-switching est consistante selon (Deaecto, Fioravanti, Geromel, 2013, théorème 1).

Notons que dans le cas linéaire, sans perturbation, c'est-à-dire $w_{k}=0$, et pour toute loi de commutation maintenue constante $\sigma(k)=i$, le coût optimal de $\sum_{k \in \mathbb{N}} x_{k}^{\prime} E_{i}^{\prime} E_{i} x_{k}$ sous la dynamique $x_{k+1}=A_{i} x_{k}$ est donné par $x_{0}^{\prime} \tilde{P}_{i} x_{0}$, avec $\tilde{P}_{i}=\tilde{P}_{i}^{\prime} \geq 0_{n}$ qui peut être définie de deux manières équivalentes :

- solution de l'équation de Lyapunov $A_{i}^{\prime} \tilde{P}_{i} A_{i}-\tilde{P}_{i}+E_{i}^{\prime} E_{i}=0_{n}$.

- solution du problème d'optimisation

$$
\min _{\tilde{P}_{i}} \operatorname{trace}\left(\tilde{P}_{i}\right)
$$

sous la contrainte

$$
\left[\begin{array}{ccc}
\tilde{P}_{i} & \star & \star \\
\tilde{P}_{i} A_{i} & \tilde{P}_{i} & \star \\
E_{i} & 0 & I_{p}
\end{array}\right]>0
$$

Cette remarque est au cœur de l'extension de la consistance au cas non linéaire tout en restant compatible avec celle du cas linéaire. La consistance s'appuie principalement sur le fait que la contrainte (16) est un cas particulier de la contrainte (14) en considérant un cas particulier de la matrice de Metzler $\Pi$.

Le théorème 4 résout le problème 2 dans le cadre de la performance (5).

THÉORÈME 4. - Soit le système (1) tel que l'entrée de perturbation est bornée en norme $\left(\sum_{k \in \mathbb{N}} w_{k}^{\prime} w_{k}<+\infty\right)$ et la condition initiale $x_{0}=0$. S'il existe des matrices symétriques définies positives $P_{i}, \forall i \in \mathscr{I}_{N}$, et une matrice de Metzler $\Pi \in \mathscr{M}$ et un scalaire $\rho \in \mathbb{R}^{+}$solution du problème d'optimisation

$$
\min _{P_{i}, \Pi} \rho,
$$

sous les contraintes

$$
\left[\begin{array}{cccc}
P_{i} & \star & \star & \star \\
0 & \rho I_{r} & \star & \star \\
(P)_{p, i} A_{i} & (P)_{p, i} H_{i} & (P)_{p, i} & \star \\
E_{i} & G_{i} & 0 & I_{p}
\end{array}\right]>0, \quad \forall i \in \mathscr{I}_{N},
$$

alors la loi de commutation définie par $\sigma_{s}\left(x_{k}\right)=\arg \min _{i \in \mathscr{I}_{N}} x_{k}^{\prime} P_{i} x_{k}$ stabilise globalement, uniformément et asymptotiquement le système (1), et le gain $\mathscr{L}_{2}$, associé à la fonction coût (5), est $\gamma \leq \sqrt{\rho}$ qui maximise le rejet de perturbation. De plus cette loi de commutation est consistante. 
PREUVE. - D'après (Deaecto et al., 2011, théorème 1), si les contraintes (18) sont vérifiées, alors le système (1) est globalement, uniformément et asymptotiquement stable et que l'inégalité (8) est vérifiée. L'objectif du problème d'optimisation (17) permet de sélectionner la meilleure solution parmi toutes les matrices de Metzler. La stratégie de min-switching est alors consistante selon (Deaecto, Fioravanti, Geromel, 2013, théorème 2).

L'introduction de la matrice $(P)_{p, i}$, faisant apparaitre un produit de variables (les composantes de la matrice $\Pi$ et les matrices $P_{i}$ ) dans le problème d'optimisation mène à des contraintes (14) ou (18) bilinéaires. La solution préconisée dans la littérature (Deaecto et al., 2011) et employée ici est d'utiliser la sous classe de matrices de Metlzer $\mathscr{M}^{\text {so }}$ suivante:

$$
\mathscr{M}^{\text {so }}=\left\{\begin{array}{c}
\Pi \in \mathbb{R}^{N \times N}, \forall(i, \ell) \in \mathscr{I}_{N}^{2}, \pi_{i i}=\gamma_{i} \in[0,1[, \\
\forall i \neq \ell, \pi_{\ell i} \geq 0, \sum_{\ell \in \mathscr{I}_{N} \backslash\{i\}} \pi_{\ell i}=1-\gamma_{i}
\end{array}\right\} .
$$

Le problème d'optimisation est alors sous forme LMI, en effectuant une recherche en ligne sur les paramètres $\gamma_{i}$ composant la matrice de Metzler.

Proposition 5. - Pour tous les scalaires $0 \leq \gamma_{i}<1, i \in \mathscr{I}_{N}$, les théorèmes 3 et 4 , restent valides avec le choix de la matrice de Metzler $\Pi_{i j} \in \mathscr{M}^{\text {so }}$ définie à l'équation (19), tel que $\pi_{j i}=1-\gamma_{i}$, avec $j \neq i$. La matrice $(P)_{p, i}$ prend alors la forme particulière $(P)_{p, i}=\gamma_{i} P_{i}+\left(1-\gamma_{i}\right) P_{j}$.

La démonstration de la proposition 5 est disponible dans (Deaecto et al., 2011, corolaire 2) dans le cadre du gain induit $\mathscr{L}_{2}$ et dans (Geromel et al., 2008, corolaire 1) dans celui de la performance (3).

Après avoir rappelé les informations nécessaires à la compréhension de la notion de consistance sur les systèmes linéaires, nous allons étendre cette notion dans la section suivante aux systèmes de Lur'e à temps discret.

\section{Consistance pour les systèmes de Lur'e commutés à temps discret}

Considérons la classe suivante de systèmes non linéaires à temps discret :

$$
\left\{\begin{aligned}
x_{k+1} & =A_{\sigma(k)} x_{k}+B_{\sigma(k)} \varphi_{\sigma(k)}\left(y_{k}\right), \\
y_{k} & =C_{\sigma(k)} x_{k}
\end{aligned}\right.
$$

où $x_{k} \in \mathbb{R}^{n}$ est le vecteur d'état et $y_{k} \in \mathbb{R}^{p}$ est le vecteur de sortie du système. La loi de commutation est notée $\sigma: \mathbb{N} \rightarrow \mathscr{I}_{N}$ où $N$ est le nombre de modes. Chaque mode est défini par des matrices réelles $A_{i}, B_{i}$ et $C_{i}$ de dimensions appropriées, $\forall i \in \mathscr{I}_{N}$. Les nonlinéarités $\varphi_{i}: \mathbb{R}^{p} \rightarrow \mathbb{R}^{p}$ sont supposées être décentralisées et vérifient des conditions de secteur de type cône borné (Khalil, 2002), $\forall i \in \mathscr{I}_{N}$. Cela signifie que chaque ligne $\ell \in\{1 ; \cdots ; p\}, \varphi_{i,(\ell)}\left(y_{k}\right)=\varphi_{i,(\ell)}\left(y_{k(\ell)}\right), \varphi_{i,(\ell)}(0)=0$ et $\varphi_{i,(\ell)}\left(y_{k}\right)^{\prime}\left[\varphi_{i}\left(y_{k}\right)-\Omega_{i} y_{k}\right]_{(\ell)} \leq 0$, 
$\forall y \in \mathbb{R}^{p}$, où $\Omega \in \mathbb{R}^{p \times p}$ est une matrice diagonale semi-définie positive. Cela implique les conditions de secteur généralisées :

$$
\varphi_{i}^{\prime}\left(y_{k}\right) S_{i}\left(\varphi_{i}\left(y_{k}\right)-\Omega_{i} y_{k}\right) \leq 0,
$$

où $S_{i} \in \mathbb{R}^{p \times p}$ sont des matrices diagonales définies positives quelconques, $\forall i \in \mathscr{I}_{N}$.

Généralement la loi de commutation $\sigma(\cdot)$ est recherchée de manière à assurer uniquement la stabilité asymptotique globale ou locale pour le système (20), voir par exemple (Gonzaga, 2012; Gonzaga et al., 2012a). Ici nous introduisons une contrainte supplémentaire en attribuant un poids quadratique à chaque mode, de manière à ce que la loi de commutation garantisse d'une part la stabilité asymptotique globale du système (20), mais aussi minimise la fonction coût

$$
\mathscr{J}_{\sigma}\left(x_{0}\right)=\sum_{k=0}^{+\infty} x_{k}^{\prime} Q_{\sigma(k)} x_{k},
$$

où les matrices $Q_{i}$ sont connues, symétriques et définies positives, $\forall i \in \mathscr{I}_{N}$ et $x_{0} \in \mathbb{R}^{n}$ est la condition initiale considérée. Ces pondérations sont fixées et définies en fonction de l'application étudiée.

Comme il a été souligné dans l'introduction, à cause de la présence des nonlinéarités dans le modèle de Lur'e à temps discret, les méthodes fondées sur les fonctions de Lyapunov ne peuvent pas mener à la performance optimale, mais uniquement à des majorants de celle-ci. Nous définissons ainsi dans la proposition suivante la classe des majorants que l'on va considérer.

Proposition 6. - Voir (Louis et al., $2015 a$; 2015b). Pour la classe des systèmes définis par (20) et une loi de commutation maintenue constante $\sigma=\ell$, s'il existe une matrice symétrique définie positive $P_{\ell} \in \mathbb{R}^{n \times n}$ et une matrice diagonale définie positive $S_{\ell} \in \mathbb{R}^{p \times p}$ qui soient solution du problème d'optimisation

$$
\underset{P_{\ell}, S_{\ell}}{\min } \operatorname{Trace}\left(P_{\ell}\right)
$$

sous les contraintes

$$
\left[\begin{array}{cc}
A_{\ell}^{\prime} P_{\ell} A_{\ell}-P_{\ell}+Q_{\ell} & \star \\
B_{\ell}^{\prime} P_{\ell} A_{\ell}+S_{\ell} \Omega_{\ell} C_{\ell} & B_{\ell}^{\prime} P_{\ell} B_{\ell}-2 S_{\ell}
\end{array}\right]<0 .
$$

alors l'origine du mode $\ell$ est globalement asymptotiquement stable et un majorant (le plus petit dans cette classe) de la fonction coût (22) est donné par $\overline{\mathscr{J}}_{\ell}=x_{0}^{\prime} P_{\ell} x_{0}$, où $x_{0}$ est la condition initiale.

Dans la suite, si la contrainte (24) du problème d'optimisation (23) n'est pas faisable, nous poserons par abus de notation $\overline{\mathscr{J}}_{\ell}=+\infty$. Nous proposons la preuve suivante de la proposition 6.

PREUVE. - En post-multipliant l'inégalité (24) par $\left(x_{k}^{\prime} \varphi_{\ell}^{\prime}\left(C_{\ell} x_{\ell}\right)\right)^{\prime}$ et en la prémultipliant par sa transposée, nous obtenons

$$
V_{\ell}\left(x_{k+1}\right)-V_{\ell}\left(x_{k}\right)<-x_{k}^{\prime} Q_{\ell} x_{k}+2 \varphi_{\ell}^{\prime}\left(y_{k}\right) S_{\ell}\left(\varphi_{\ell}\left(y_{k}\right)-\Omega_{\ell} y_{k}\right) .
$$


avec $V_{\ell}\left(x_{k}\right)=x_{k}^{\prime} P_{\ell} x_{k}$ une fonction de Lyapunov quadratique. Comme $Q_{\ell}$ est une matrice symétrique définie positive et à cause de la condition de secteur (21), l'inégalité (25) implique que le mode $\ell$ est globalement asymptotiquement stable et que $\lim V_{\ell}\left(x_{k}\right)=0$. Nous en déduisons, à partir de l'inégalité (25) le majorant de la fonction coût (22) :

$$
\overline{\mathscr{J}}_{\ell}\left(x_{0}\right)=V_{\ell}\left(x_{0}\right)>\sum_{k=0}^{+\infty} x_{k}^{\prime} Q_{\ell} x_{k}=\mathscr{J}_{\ell}\left(x_{0}\right) .
$$

Comme dans le cas linéaire, l'ensemble $\mathscr{S}$ est défini comme contenant toutes les lois de commutations qui sont un retour d'état, c'est-à-dire dont $\sigma(k)$ est une fonction de $x_{k}$ et qui assurent la stabilité asymptotique globale du système (20). De la même manière, nous définissons l'ensemble $\mathscr{C}$ contenant les $N$ lois de commutation maintenues constantes, c'est-à-dire telles que $\forall k \in \mathbb{N}, \sigma(k)=i \in \mathscr{I}_{N}$. $\mathscr{C}$ peut être décomposé en deux sous-ensembles: $\mathscr{L}$ contenant les lois de commutation constantes pour lesquelles l'inégalité modale (24) est faisable et $\overline{\mathscr{L}}$ les autres lois de commutation constantes (en d'autres termes les conditions modales (24) ne sont pas faisables et par abus de notation sont associés à des majorants infinis $\left.\overline{\mathscr{J}}_{\ell}=+\infty\right)$. Notons que pour le cas linéaire, il y a équivalence entre stabilité du mode et faisabilité de l'inégalité (16). Ce n'est plus le cas entre stabilité du mode de Lur'e et faisabilité de l'inégalité (24), d'où la distinction ici.

La présence de la non-linéarité dans les modèles de Lur'e à temps discret conduit à considérer des majorants définis dans la proposition 6. Ainsi, sans pouvoir déterminer exactement la valeur du coût, la définition de la consistance en linéaire faisant intervenir le coût optimal de chaque mode ne peut pas être directement utilisée. La consistance d'une loi de commutation se définit de la manière suivante dans notre cadre non linéaire, tout en restant valide pour le cadre linéaire.

DÉFInITION 7. - Considérons le système commuté (20). Une stratégie de commutation particulière $\sigma_{s}(\cdot)$ est consistante si elle appartient à l'ensemble $\mathscr{S}$ et garantit $\forall \sigma \in \mathscr{C}$ et $\forall x_{0} \in \mathbb{R}^{n}$ que

$$
\overline{\mathscr{J}}_{\sigma_{s}}\left(x_{0}\right) \leq \overline{\mathscr{J}}_{\sigma}\left(x_{0}\right),
$$

où $\overline{\mathscr{J}}_{\sigma}\left(x_{0}\right)$ sont définis par la proposition 6.

Le problème qui nous intéresse est décrit de la manière suivante :

Problème 8. - Construire une loi de commutation $\sigma_{s}$ et un majorant quadratique $\overline{\mathscr{J}}_{\sigma_{s}}\left(x_{0}\right)$ de la fonction coût (22), aussi petit que possible, tel que la propriété de consistance de la définition 7 soit satisfaite.

Le théorème suivant propose une solution à ce problème.

THÉORÈMe 9. - Pour la classe des systèmes définis par (20) et une fonction coût (22), s'il existe une matrice de Metzler $\Pi \in \mathscr{M}$, des matrices symétriques définies 
positives $P_{i} \in \mathbb{R}^{n \times n}$ et des matrices diagonales définies positives $S_{i} \in \mathbb{R}^{p \times p}$, telles que $\forall i \in \mathscr{I}_{N}$

$$
\min _{P_{i}, S_{i}, \Pi}\left(\min _{i \in \mathscr{I}_{N}} \operatorname{trace}\left(P_{i}\right)\right),
$$

sous les contraintes

$$
\left[\begin{array}{cc}
A_{i}^{\prime}(P)_{p, i} A_{i}-P_{i}+Q_{i} & \star \\
B_{i}^{\prime}(P)_{p, i} A_{i}+S_{i} \Omega_{i} C_{i} & B_{i}^{\prime}(P)_{p, i} B_{i}-2 S_{i}
\end{array}\right]<0,
$$

alors la stratégie de min-switching

$$
\sigma\left(x_{k}\right)=\arg \min _{i \in \mathscr{I}_{N}} x_{k}^{\prime} P_{i} x_{k}
$$

garantit que l'origine du système (20) est globalement asymptotiquement stable. De plus, un majorant de la fonction coût (22) est donné par $\overline{\mathscr{J}}_{\sigma_{s}}=V_{\min }\left(x_{0}\right)$, où $x_{0}$ est la condition initiale et

$$
V_{\min }\left(x_{k}\right)=\min _{i \in \mathscr{I}_{N}} x_{k}^{\prime} P_{i} x_{k},
$$

est une fonction de Lyapunov composite. La stratégie de min-switching (30) est consistante selon la définition 7.

Pour prouver que la loi de commutation (30) est consistante, il est nécessaire de comparer le majorant induit par le problème d'optimisation (28) avec le majorant donné par l'étude de chaque mode indépendamment les uns des autres.

PREUVE. - Cette preuve est décomposée en deux parties. La première partie donne un majorant de la fonction coût considérée en association de la stratégie de minswitching. La seconde partie justifie que le majorant donné par (30) est consistant.

Etape 1 : en introduisant $m_{k}=\left(x_{k}^{\prime} \varphi_{i}^{\prime}\left(C_{i} x_{k}\right)\right)^{\prime}$, la fonction de Lyapunov (31) vérifie l'inégalité

$$
V_{\min }\left(x_{k+1}\right)=\min _{\lambda_{j} \in[0,1], \lambda_{1}+\cdots+\lambda_{N}=1} \sum_{j \in \mathscr{I}_{N}} \lambda_{j} m_{k}^{\prime}\left[\begin{array}{cc}
A_{i}^{\prime} P_{j} A_{i} & \star \\
B_{i}^{\prime} P_{j} A_{i} & B_{i}^{\prime} P_{j} B_{i}
\end{array}\right] m_{k} .
$$

En supposant vérifiée la condition de secteur (21) et en considérant une matrice de Metzler $\Pi \in \mathscr{M}$, nous avons

$$
V_{\min }\left(x_{k+1}\right) \leq m_{k}^{\prime}\left[\begin{array}{cc}
A_{i}^{\prime}(P)_{p, i} A_{i}+Q_{i} & \star \\
B_{i}^{\prime}(P)_{p, i} A_{i}+S_{i} \Omega_{i} C_{i} & B_{i}^{\prime}(P)_{p, i} B_{i}-2 S_{i}
\end{array}\right] m_{k}
$$

Pour obtenir l'équation (29), nous soustrayons la valeur $V_{\min }\left(x_{k}\right)=x_{k} P_{i^{*}} x_{k}$ et $d u$ fait que le mode $i^{*}=\underset{j \in \mathscr{I}_{N}}{\operatorname{argmin}} x_{k}^{\prime} P_{j} x_{k}$ est défini comme actif à l'instant $k$,

$$
V_{\min }\left(x_{k+1}\right)-V_{\min }\left(x_{k}\right) \leq m_{k}^{\prime}\left[\begin{array}{lc}
A_{i^{*}}^{\prime}(P)_{p, i^{*}} A_{i^{*}}-P_{i^{*}}+Q_{i^{*}} & \star \\
B_{i^{*}}^{\prime}(P)_{p, i^{*}} A_{i^{*}}+S_{i^{*}} \Omega_{i^{*}} C_{i^{*}} & B_{i^{*}}^{\prime}(P)_{p, i^{*}} B_{i^{*}}-2 S_{i^{*}}
\end{array}\right] m_{k} .
$$


Cette dernière inégalité peut se réécrire en

$$
V_{\min }\left(x_{k+1}\right)-V_{\min }\left(x_{k}\right) \leq-x_{k}^{\prime} Q_{i^{*}} x_{k} .
$$

Grâce au fait que $Q_{i^{*}}$ est symétrique et définie positive, l'inégalité (35) implique que le système (20) soit globalement asymptotiquement stable. Cela signifie que $\lim _{k \rightarrow+\infty} V_{\min }\left(x_{k}\right)=0$. La loi de commutation de type min-switching (30), notée $\sigma_{s}$ appartient à l'ensemble $\mathscr{S}$. Grâce à l'inégalité (36) le majorant de la fonction coût (22) est

$$
\begin{aligned}
& \sum_{k=0}^{+\infty}\left(V_{\min }\left(x_{k+1}\right)-V_{\min }\left(x_{k}\right)\right)<-\mathscr{J}_{\sigma_{s}}\left(x_{0}\right), \\
& \overline{\mathscr{J}}_{\sigma_{s}}\left(x_{0}\right)=V_{\min }\left(x_{0}\right)>\mathscr{J}_{\sigma_{s}}\left(x_{0}\right) .
\end{aligned}
$$

Etape 2 : pour prouver que la stratégie de type min-switching $\sigma_{s}$ est consistante dans le sens donné par la définition 7, nous supposons qu'au moins un des modes, noté $\ell$ appartient à $\mathscr{L}$. En d'autres termes, le sous-système caractérisé par $\left\{A_{\ell} ; B_{\ell} ; C_{\ell} ; \Omega_{\ell}\right\}$ est solution du problème d'optimisation (23). Cette hypothèse est nécessaire pour avoir l'ensemble $\mathscr{L}$ non vide.

Sélectionnons $\Pi_{\ell} \in \mathscr{M}$ avec des composantes nulles, à l'exception de $\forall j \in \mathscr{I}_{N}$, $\pi_{\ell j}=1$, tel que $(P)_{p, j}=P_{\ell}$. Les contraintes (29) deviennent alors $\forall i \in \mathscr{I}_{N} \backslash\{\ell\}$,

$$
\left[\begin{array}{cc}
A_{i}^{\prime} P_{\ell} A_{i}-P_{i}+Q_{i} & \star \\
B_{i}^{\prime} P_{\ell} A_{i}+S_{i} \Omega_{i} C_{i} & B_{i}^{\prime} P_{\ell} B_{i}-2 S_{i}
\end{array}\right]<0
$$

et l'inégalité (24) pour $i=\ell . \forall i \in \mathscr{I}_{N} \backslash\{\ell\}$, les matrices $P_{i}$ et $S_{i}$ sont arbitrairement choisies et fixées de telle manière que $P_{i}>P_{\ell}$. Ainsi, comme $P_{\ell}$ vérifie toujours (24), il est toujours possible de trouver des valeurs pour ces matrices afin de satisfaire (38). Ainsi, grâce à la structure de la matrice $\Pi_{\ell}$, les contraintes (29) se réécrivent sous la forme (24). Nous rappelons que

$$
\overline{\mathscr{J}}_{\sigma_{s}}\left(x_{0}\right)=V_{\min }\left(x_{0}\right)=\min _{i \in \mathscr{I}_{N}} x_{0}^{\prime} P_{i} x_{0},
$$

où les $P_{i}$ sont solutions du problème d'optimisation (28) avec $\Pi \in \mathscr{M}$. En considérant la structure de la matrice de Metzler $\Pi_{\ell}$, nous avons :

$$
\overline{\mathscr{J}}_{\sigma_{s}}\left(x_{0}\right) \leq \min _{i \in \mathscr{I}_{N}} x_{0}^{\prime} P_{i} x_{0},
$$

avec $P_{i}$ sont solutions du problème d'optimisation (28) avec $\Pi_{\ell} \in \mathscr{M}$. Dans ce cas, nous montrons que $P_{i}\left(>P_{\ell}\right), \forall i \neq \ell$ convient et

$$
\overline{\mathscr{J}}_{\sigma_{s}}\left(x_{0}\right) \leq x_{0}^{\prime} P_{\ell} x_{0}=\overline{\mathscr{J}}_{\ell}\left(x_{0}\right), \quad \forall \ell \in \underline{\mathscr{L}} .
$$

Finalement, comme les autres lois de commutation constantes appartenant à $\overline{\mathscr{L}}$ sont associées à des majorants infinis, l'inégalité (41) est vérifiée $\forall \ell \in \mathscr{C}$. Cela termine la preuve. 
En s'inspirant de la littérature (Geromel et al., 2011; Deaecto, Souza, Geromel, 2013 ; Deaecto, Fioravanti, Geromel, 2013), nous considérons la sous-classe des matrices de Metzler $\mathscr{M}^{\text {so }}$ définie par la relation (19). Même si cette approche est conservative, elle permet d'obtenir des contraintes de type LMI (aisément manipulables numériquement) pour la proposition 10 dépendant uniquement de $N$ scalaires à choisir. Nous nous inspirons de cette littérature pour proposer une procédure de recherche en ligne sur les scalaires $\gamma_{i}$ qui est utilisée pour trouver la meilleure solution à l'aide de $\mathscr{M}^{\text {so }}$ en se fondant sur la proposition 10.

Proposition 10. - Pour la classe des systèmes définis par (20), pour la fonction coût (22) et pour des scalaires fixés $0 \leq \gamma_{i}<1$, s'il existe des matrices symétriques définies positives $P_{i}$ et des matrices diagonales définies positives $S_{i}$, avec $i \in \mathscr{I}_{N}$, tel que

$$
\min _{P_{i}, S_{i}}\left(\min _{i \in \mathscr{I}_{N}} \operatorname{trace}\left(P_{i}\right)\right)
$$

sous les contraintes $\forall(i, j) \in \mathscr{I}_{N}^{2}, i \neq j$,

$$
\left[\begin{array}{cc}
A_{i}^{\prime} \Delta_{i j} A_{i}-P_{i}+Q_{i} & \star \\
B_{i}^{\prime} \Delta_{i j} A_{i}+S_{i} \Omega_{i} C_{i} & B_{i}^{\prime} \Delta_{i j} B_{i}-2 S_{i}
\end{array}\right]<0,
$$

avec $\Delta_{i j}=\gamma_{i} P_{i}+\left(1-\gamma_{i}\right) P_{j}$, alors la stratégie (30) garantit que l'origine est globalement asymptotiquement stable. Un majorant de la fonction coût (22) est donné par $\overline{\mathscr{J}}_{\sigma_{s}}\left(x_{0}\right)=V_{\min }\left(x_{0}\right)$, avec $V_{\min }(\cdot)$ défini par l'équation (31). La stratégie minswitching considérée est consistante au sens de la définition 7.

PREUVE. - Les scalaires $\gamma_{i}=\pi_{i i}$ sont choisis tels que $\Pi \in \mathscr{M}^{\text {so }}$. Ainsi, en multipliant l'équation (43) par $\pi_{j i}$ et en sommant sur l'ensemble $j \in \mathscr{I}_{N}$, tel que $j \neq i$, nous obtenons, avec $D_{i}=\pi_{i i} P_{i}+\sum_{j \in \mathscr{I}_{N}, j \neq i} \pi_{j i} P_{j}$

$$
\left(1-\gamma_{i}\right)\left[\begin{array}{cc}
A_{i}^{\prime} D_{i} A_{i}-P_{i}+Q_{i} & \star \\
B_{i}^{\prime} D_{i} A_{i}+S_{i} \Omega_{i} C_{i} & B^{\prime} D_{i} B-2 S_{i}
\end{array}\right]<0 .
$$

En multipliant cette dernière inégalité par $\left(1-\gamma_{i}\right)^{-1}, \forall i \in \mathscr{I}_{N}$, nous obtenons une solution particulière de l'inégalité (29). En prenant les cas particuliers $\gamma_{i}=0$ ou $\gamma_{i}=1$, nous retrouvons les cas particuliers mentionnés dans la preuve 3. En suivant la preuve 3, nous établissons que la stratégie de min-switching donnée par la proposition 10 est consistante. 
Exemple académique : cet exemple illustre numériquement cette propriété de consistance. Il est choisi de dimension deux afin de pouvoir comparer diverses trajectoires dans le plan. Nous considérons le système (20) avec :

$$
\begin{gathered}
A_{1}=\left[\begin{array}{cc}
0,9 & 0 \\
0,4 & -0,72
\end{array}\right], A_{2}=\left[\begin{array}{cc}
-0,58 & -0,8 \\
0 & -0,8
\end{array}\right], x_{0}=\left(\begin{array}{c}
-4 \\
5
\end{array}\right) \\
B_{1}=-\left[\begin{array}{l}
0,5 \\
0,2
\end{array}\right], B_{2}=\left[\begin{array}{l}
0,2 \\
0,5
\end{array}\right], C_{1}=\left[\begin{array}{ll}
0,6 & 0,24
\end{array}\right], \\
C_{2}=\left[\begin{array}{ll}
0,4 & 1,1
\end{array}\right], \varphi_{1}\left(y_{k}\right)=\frac{\Omega_{1} y_{k}}{2}\left(1+\cos \left(2 y_{k}\right)\right), \\
\varphi_{2}\left(y_{k}\right)=\frac{\Omega_{2} y_{k}}{2}\left(1-\sin \left(5,5 y_{k}\right)\right), \Omega_{1}=0,6, \Omega_{2}=1,2 .
\end{gathered}
$$

Le tableau suivant indique les majorants des fonctions coût pour la condition initiale $x_{0}$ et différentes valeurs $q_{i}$, tel que $Q_{i}=q_{i} I_{n}$ et $i \in \mathscr{I}_{N} \cdot \bar{J}_{i}$ (resp. $\overline{\mathscr{J}}_{\sigma_{s}}$ ) désignent le majorant de la fonction coût donné pour le mode $i$ (resp. par la loi de commutation min-switching (30), voir proposition 10). Une recherche en ligne est effectuée sur $\gamma_{i}$, tel que $0 \leq \gamma_{i} \leq 1$ avec un pas de 0.1 .

\begin{tabular}{|c|c|c|c|c|c|c|c|}
\hline$q_{1}$ & $q_{2}$ & $\overline{\mathscr{J}}_{1}$ & $\overline{\mathscr{J}}_{2}$ & $\overline{\mathscr{J}}_{\sigma_{s}}$ & $\mathscr{J}_{1}$ & $\mathscr{J}_{2}$ & $\mathscr{J}_{\sigma_{s}}$ \\
\hline 1 & 1 & 234 & 267 & 96 & 138 & 91 & 70 \\
\hline 4 & 1 & 939 & 267 & 222 & 553 & 91 & 193 \\
\hline 1 & 4 & 234 & 1070 & 234 & 138 & 364 & 138 \\
\hline
\end{tabular}

Notons que le majorant $\bar{J}_{\sigma_{s}}$ donné par la proposition 10 est toujours plus petit ou égal que le coût obtenu par chaque mode séparément $\overline{\mathscr{J}}_{i}, \forall i \in \mathscr{I}_{2}$. Cependant nous ne garantissons pas que la valeur exacte du coût vérifie la propriété de consistance à cause de la non-linéarité: par exemple, pour $\left(q_{1}, q_{2}\right)=(4,1), \mathscr{J}_{2}<\mathscr{J}_{\sigma_{\mathrm{s}}}$. L'écart entre les coûts exacts $\mathscr{J}_{i}$ et le majorant $\overline{\mathscr{J}}_{i}$ peut être important. Il faut néanmoins rappeler que notre approche reste valable pour toute condition initiale et non uniquement pour cette valeur de $x_{0}$, ainsi que pour toute non-linéarité vérifiant la condition de secteur (21). Ce qui peut expliquer en grande partie cet écart. Les trajectoires considérées sont représentées sur la figure 1 où les zones d'activation des modes 1 et 2 sont colorées de manières différentes.

Nous venons de prouver que la stratégie de min-switching (30) garantit un meilleur majorant quadratique de la fonction coût (22) qu'en considérant un mode isolé. La section suivante s'attache à appliquer ce résultat à l'implémentation d'une synthèse de commande de type synthèse à temps continu (STC) avec un échantillonnage non uniforme pour les systèmes de Lur'e à temps continu. L'idée est d'attribuer à chaque période d'échantillonnage une pondération quadratique qui reflète le fait que l'on veut éviter d'utiliser les faibles périodes d'échantillonnages. 


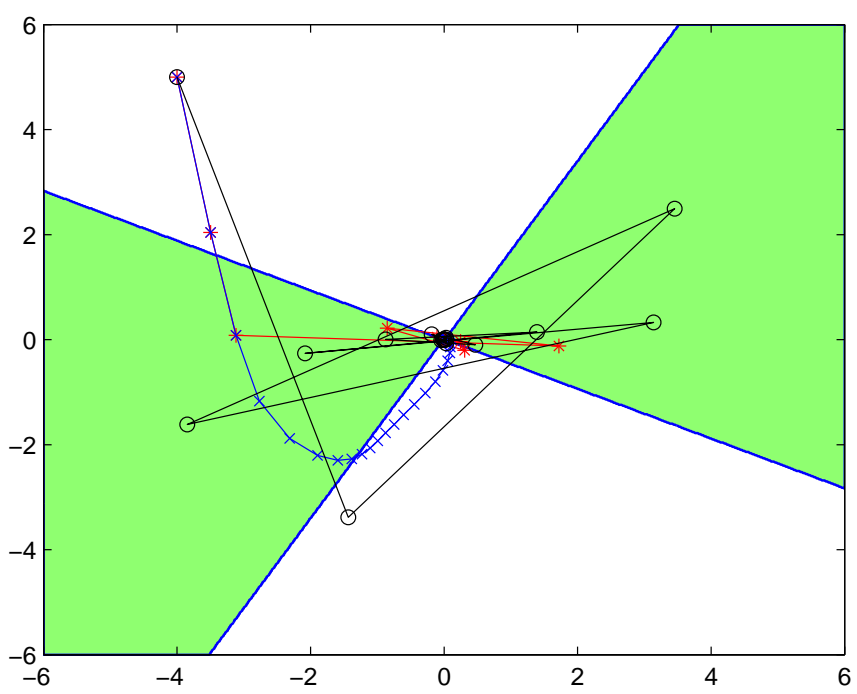

Figure 1. Les zones blanches et vertes représentent les zones d'activation des modes 1 et 2 . Elles sont obtenues par la proposition 10 avec $\gamma_{1}=0,2, \gamma_{2}=1$ et $q_{1}=q_{2}=1$. Les lignes bleues et noires sont les trajectoires des modes 1 et 2 , où les croix et cercles indiquent les échantillons. La ligne rouge représente la trajectoire commutée, avec les étoiles pour les échantillons

\section{Application : synthèse STC à échantillonnage non uniforme}

Considérons le système non linéaire à temps continu

$$
\left\{\begin{array}{l}
\dot{x}(t)=A_{c} x(t)+B_{c} \varphi_{c}(y(t))+F_{c} u(t) \\
y(t)=C_{c} x(t)
\end{array}\right.
$$

où $x(t) \in \mathbb{R}^{n}$ est le vecteur d'état, $y(t) \in \mathbb{R}^{p}$ le vecteur de sortie et $u(t) \in \mathbb{R}^{m}$ l'entrée de commande. La non-linéarité $\varphi_{c}: \mathbb{R}^{p} \rightarrow \mathbb{R}^{p}$ est supposée décentralisée et satisfaisant la condition de secteur $\varphi_{c}^{\prime}(y(t)) S\left(\varphi_{c}(y(t))-\Omega_{c} y(t)\right) \leq 0$, où $\Omega_{c}$ et $S \in \mathbb{R}^{p \times p}$ sont des matrices diagonales et définies positives. Le contrôleur est défini par $u(t)=K x(t)+$ $\Gamma \varphi_{c}(y(t))$, avec $K$ et $\Gamma$ supposés donnés par une loi de synthèse en temps continu. Le problème qui nous intéresse est le suivant.

Problème 11. - Comment choisir dans une famille finie de périodes $T_{i},\left(i \in \mathscr{I}_{N}\right)$ pour faire la synthèse STC à échantillonnage non uniforme et assurer la stabilité asymptotique globale du système (45) à l'aide de

$$
u(t)=u_{k}, \forall t \in[k T ;(k+1) T[, k \in \mathbb{N} .
$$


Pour fabriquer cette commande numérique, une discrétisation du système (45) est nécessaire. La non-linéarité ne permet pas d'obtenir une expression analytique exacte du discrétisé. Nous choisissons alors une approximation du type Euler explicite qui permet de conserver en temps discret la structure d'une interconnexion de Lur'e. Pour une famille de périodes d'échantillonnage $T_{i}$, l'approximation d'Euler en boucle fermée est donnée par le système commuté :

$$
\left\{\begin{aligned}
x_{k+1} & =\left(I_{2}+T_{\sigma(k)}\left(A_{c}+F_{c} K\right)\right) x_{k}+T_{\sigma(k)}\left(B_{c}+F_{c} \Gamma\right) \varphi_{c}\left(y_{k}\right), \\
y_{k} & =C_{c} x_{k}
\end{aligned}\right.
$$

Ce système est un cas particulier de système commuté (20) pour lequel la loi de commutation détermine la période à appliquer. Par identification $\varphi_{i}\left(y_{k}\right)=\varphi_{c}\left(y_{k}\right), A_{i}=$ $I_{2}+T_{i}\left(A_{c}+F_{c} K\right), B_{i}=T_{i}\left(B_{c}+F_{c} \Gamma\right)$ et $C_{i}=C_{c}, \forall i \in \mathscr{I}_{N}$. Les pondérations $Q_{i}$ sont définies telles que $\forall(i, j) \in \mathscr{I}_{N}^{2}, Q_{i}>Q_{j}$ si $T_{j}>T_{i}$.

Le théorème 9 assure la consistance de la stratégie min-switching vis-à-vis du majorant du coût (22) pour le modèle approché d'Euler. C'est-à-dire que cette stratégie mène à la réduction du majorant ou bien dans le pire des cas au majorant obtenu en considérant une seule période d'échantillonnage. Comme plusieurs périodes d'échantillonnage sont utilisées, nous définissons une période d'échantillonnage moyenne $T_{\text {moy }}$ comme la période virtuelle représentant la valeur moyenne des périodes, pondérées par leur fréquence d'activation. Elle est évaluée a posteriori. L'exemple académique suivant illustre le bénéfice d'une telle stratégie commutée.

Exemple : La méthode est appliquée sur le système à temps continu (45) avec

$$
\begin{gathered}
A_{c}=\left[\begin{array}{cc}
-6 & -1 \\
2 & -3,72
\end{array}\right], B_{c}=\left[\begin{array}{l}
0,5 \\
0,5
\end{array}\right], C_{c}=\left[\begin{array}{ll}
1 & 0,4
\end{array}\right], \\
\varphi_{c}\left(y_{k}\right)=\frac{\Omega_{c} y_{k}}{2}\left(1+\cos \left(2 y_{k}\right)\right), \Omega_{c}=0,6, x_{0}=\left(\begin{array}{l}
60 \\
60
\end{array}\right) .
\end{gathered}
$$

L'esprit de la méthode STC est que l'échantillonnage du contrôleur continu est d'autant meilleur candidat que la période d'échantillonnage est suffisamment faible. Les gains $K$ et $\Gamma$ sont obtenus à l'aide de la méthode (Castelan et al., 2008) : $K=$ $[-0,0471-0,1045]$ et $\Gamma=-0.1646$. Nous supposons que l'implémentation se fait sur un système embarqué pour lequel une période d'échantillonnage faible est associée à une pondération importante. Prenons l'exemple $T_{1}=0,2$ et $T_{2}=0,4$ et associons les coûts (22) donnés par $Q_{1}=10 I_{2}$ et $Q_{2}=I_{2}$. Le problème d'optimisation (23) mène à $\overline{\mathscr{J}}_{1}=94059$ et $\overline{\mathscr{J}}_{2}=+\infty$ (le discrétisé d'Euler avec $T_{2}$ est instable). En considérant la stratégie de min-switching, on obtient alors $\overline{\mathscr{J}}_{\sigma_{s}}=93406$. Ce coût n'a été que peu amélioré par rapport à l'utilisation de $T_{1}$ uniquement. Néanmoins, sur cet exemple, la stratégie de min-switching utilisant $T_{2}$, la période d'échantillonnage moyenne a été améliorée $T_{\text {moy }}=0,32$ (voir la figure 2 ).

Pour montrer l'amélioration de cette technique en comparaison de l'utilisation de la période d'échantillonnage moyenne comme seule période d'échantillonnage, nous 


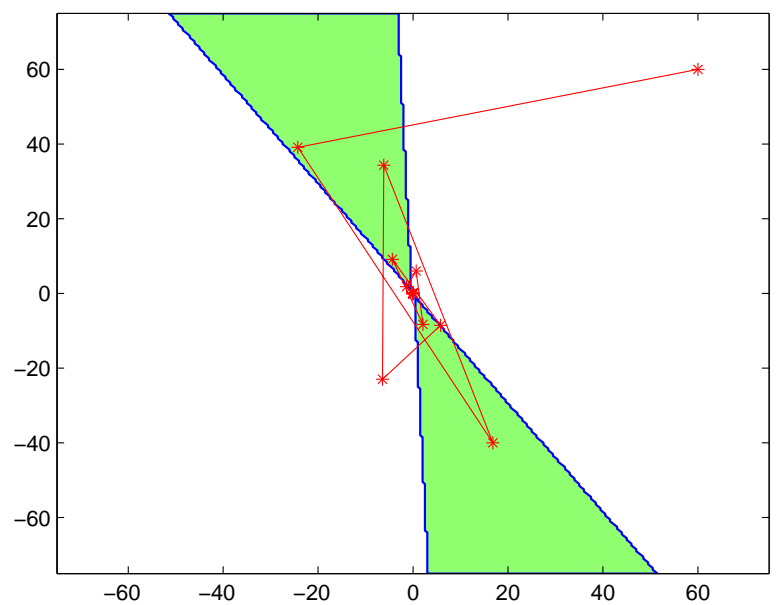

Figure 2. Les zones d'activation des modes 1 et 2 sont respectivement de couleur blanche et verte. Elles ont été obtenues à l'aide de la proposition 10 avec $\gamma_{1}=0,5$ et $\gamma_{2}=0,4$. La ligne rouge représente la trajectoire commutée et les étoiles les échantillons

introduisons la matrice $Q_{\text {moy }}=4,6 I_{2}$ qui est obtenue comme combinaison linéaire de $Q_{1}$ et $Q_{2}$ avec les pondérations identiques à celles de $T_{1}$ et $T_{2}$ pour obtenir $T_{\text {moy }}$. Le majorant donné par le problème d'optimisation (23) relatif à $T_{\text {moy }}$ vaut $\overline{\mathscr{J}}_{\text {moy }}=$ 131870. La stratégie de min-switching a donc réduit ce coût de $41 \%$.

La méthode introduite dans cet article n'assure pas la stabilité du système original à temps continu. Cependant nous illustrons cette stabilité en vérifiant la convergence de la trajectoire sur les figures 3 et 4 . Pour offrir des garanties théoriques sur la stabilité du système (45) des contraintes supplémentaires doivent être ajoutées à la méthode présentée ici. Ce problème a été étudié dans la littérature pour les systèmes non linéaires en considérant un échantillonnage uniforme (Nešić, Teel, Kokotović, 1999; Nešić, Teel, 2004 ; 2006). L'extension à l'échantillonnage non uniforme reste une perspective de recherche.

\section{Conclusions et perspectives}

Dans cet article, la notion de consistance a été discutée pour une classe de systèmes non linéaires de type Lur'e en considérant un majorant quadratique de la fonction coût. Une loi de commutation consistante a été construite par rapport à cette classe de majorants, telle que le majorant soit le plus petit possible. Ce résultat est formulé comme un problème d'optimisation sous contraintes bilinéaires. Une solution faisant intervenir une recherche en ligne sur des LMI a été proposée. Un exemple académique illustre cette contribution et souligne les principales différences avec le cas linéaire. 


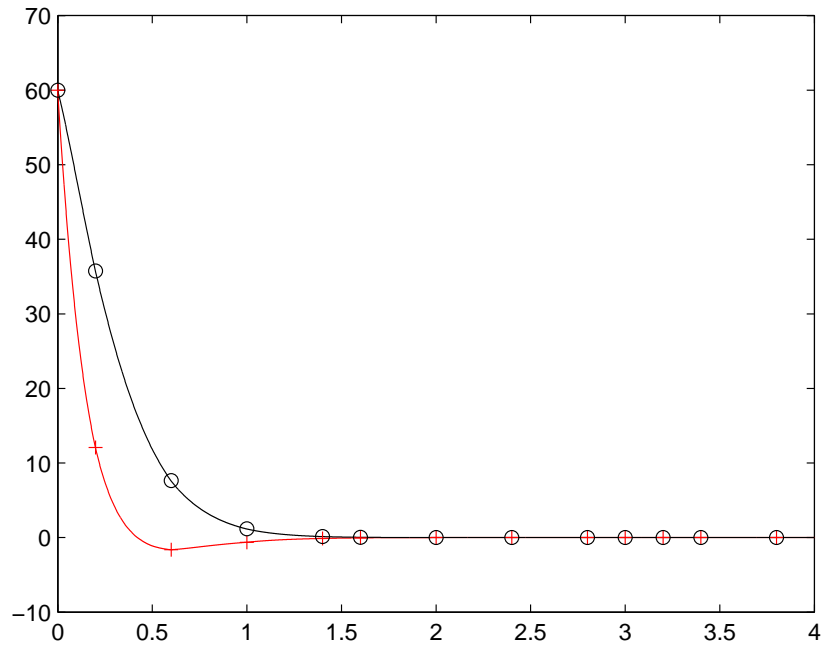

Figure 3. Les trajectoires rouge et noire représentent la première et la seconde composante de l'état en fonction du temps. Les croix et cercles représentent les échantillons

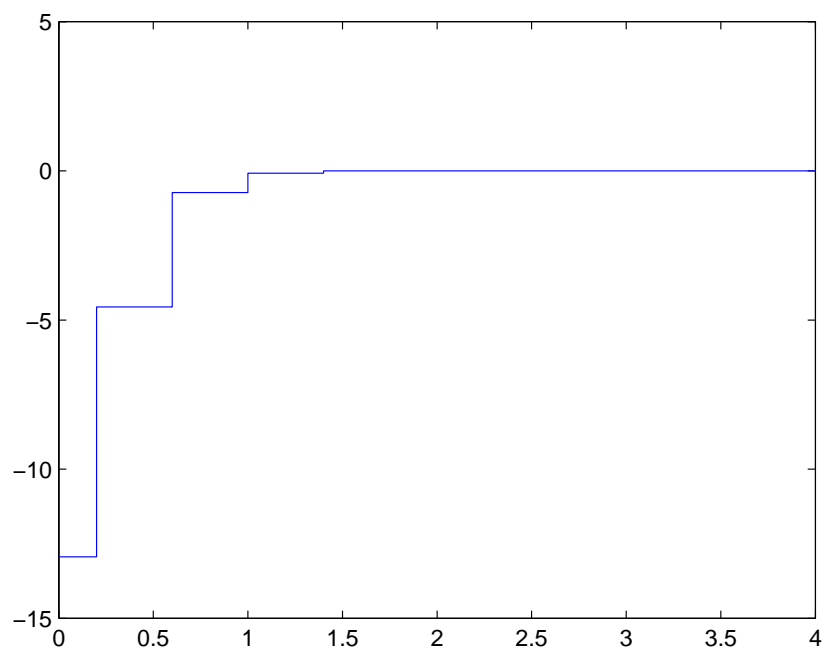

Figure 4. Commande du système (45) en fonction du temps 
Une application intéressante de ce résultat a été étudiée : l'implémentation d'une commande par données échantillonnées avec une méthode de synthèse à temps continu et un échantillonnage non uniforme. Un exemple numérique met en avant les améliorations possibles, tant pour la fonction coût que pour la période d'échantillonnage moyenne. Néanmoins nous ne proposons pas de certification de stabilité pour le système original en temps continu. Cela constitue encore un problème ouvert.

\section{Remerciements}

Ce travail a été partiellement soutenu par le septième programme-cadre de la communauté européenne (FP7/2007-2013) sous la convention de subvention $n^{\circ} 257462$ : HYCON2 Network of Excellence "Highly-Complex and Networked Control Systems", par le projet ANR COMPACS - "Computation Aware Control Systems", ANR13-BS03-004, ainsi que par la Région Lorraine.

Article soumis le 7/09/2015

Accepté le 3/02/2016

\section{Bibliographie}

Barbot J., Monaco S., Normand-Cyrot D. (1996). A sampled normal form for feedback linearization. Mathematics of Control, Signals and Systems, vol. 9, n 2, p. 162-188.

Castelan E. B., Tarbouriech S., Queinnec I. (2008). Control design for a class of nonlinear continuous-time systems. Automatica, vol. 44, no 8, p. 2034-2039.

Deaecto G., Fioravanti A., Geromel J. (2013). Suboptimal switching control consistency analysis for discrete-time switched linear systems. European Journal of Control, vol. 19, $\mathrm{n}^{\circ} 3$, p. 214-219.

Deaecto G., Geromel J., Daafouz J. (2011). Dynamic output feedback $\mathscr{H}_{\infty}$ control of switched linear systems. Automatica, vol. 47, no 8, p. 1713-1720.

Deaecto G., Souza M., Geromel J. (2013, June). State feedback switched control of discretetime switched linear systems with application to networked control. In Control Automation (MED), 21st Mediterranean Conference on, p. 877-883.

Geromel J., Colaneri P., Bolzern P. (2008, April). Dynamic output feedback control of switched linear systems. Automatic Control, IEEE Transactions on, vol. 53, nº 3, p. 720-733.

Geromel J., Deaecto G., Daafouz J. (2011, August). Suboptimal switching state feedback control consistency analysis for switched linear systems. In Proceedings of 18th IFAC World Congress, p. 5849-5854.

Geromel J., Deaecto G., Daafouz J. (2013, July). Suboptimal switching control consistency analysis for switched linear systems. Automatic Control, IEEE Transactions on, vol. 58, $\mathrm{n}^{\circ} 7$, p. $1857-1861$.

Gonzaga C. A. C. (2012). Analyse de stabilité et de performances d'une classe de systèmes non-linéaires à commutations en temps discret. Thèse de doctorat, Université de Lorraine.

Gonzaga C. A. C., Jungers M., Daafouz J. (2012a). Stability analysis and stabilisation of switched nonlinear systems. International Journal of Control, vol. 85, nº 7, p. 822-829.

Gonzaga C. A. C., Jungers M., Daafouz J. (2012b, September). Stability analysis of discrete time Lur'e systems. Automatica, vol. 48, p. 2277-2283. 
Hajiahmadi M., Schutter B. D., Hellendoorn H. (2014). Stabilization and robust $\mathscr{H}_{\infty}$ control for sector-bounded switched nonlinear systems. Automatica, vol. 50, n 10, p. 2726 - 2731.

Hsu P., Sastry S. (1987). The effect of discretized feedback in a closed loop system. In Decision and Control, 1987. 26th IEEE Conference on, vol. 26, p. 1518-1523.

Jungers M., Castelan E. B., Tarbouriech S., Daafouz J. (2011, May). Finite $L_{2}$-induced gain and $\lambda$-contractivity of discrete-time switching systems including modal nonlinearities and saturating actuator. Nonlinear Analysis: Hybrid Systems, vol. 5, nº 2, p. 289-300.

Khalil H. K. (2002). Nonlinear systems third edition. Prentice Hall.

Laila D. S., Nešić D., Astolfi A. (2005). Advanced topics in control systems theory II. In A. Loria, F. Lamnabhi-Lagarrigue, E. Panteley (Eds.),, vol. 328, p. 91-137. Springer.

Liberzon D. (2003). Systems and control: Foundations and applications. Birkhauser, Boston, MA.

Louis J. (2015). Discrétisation des systèmes de Lur'e : stabilisation et consistance. Thèse de doctorat, Université de Lorraine.

Louis J., Jungers M., Daafouz J. (2015a). Consistance des systèmes de Lur'e commutés : application à la synthèse de commande numérique avec un échantillonnage non uniforme. In Actes des Journées Doctorales Modélisation Analyse Commande des Systèmes (JDMACS). Bourges, France.

Louis J., Jungers M., Daafouz J. (2015b). Switching control consistency of switched Lur'e systems with application to digital control design with non uniform sampling. In Proceedings of the European Control Conference, p. 1748-1753. Johannes Kepler University, Linz, Austria.

Lur'e I., Postnikov V. N. (1944). On the theory of stability of control systems. In Applied Mathematics and Mechanics.

Monaco S., Normand-Cyrot D. (1985). On the sampling of a linear analytic control system. In Decision and Control, 1985 24th IEEE Conference on, vol. 24, p. 1457-1462.

Monaco S., Normand-Cyrot D. (2001). Issues on nonlinear digital control. European Journal of Control, vol. 7, p. 160-177.

Nešić D., Teel A. (2006). Stabilization of sampled-data nonlinear systems via backstepping on their euler approximate model. Automatica, vol. 42, nº 10, p. 1801-1808.

Nešić D., Teel A. R. (2004). A framework for stabilization of nonlinear sampled-data systems based on their approximate discrete-time models. IEEE Transactions on Automatic Control, vol. $49, n^{\circ} 7$, p. 1103-1122.

Nešić D., Teel A. R., Kokotović P. V. (1999). Sufficient conditions for stabilization of sampled-data nonlinear systems via discrete-time approximations. Systems \& Control Letters, vol. 38, p. 259-270.

Nešić D., Teel A. R., Sontag E. D. (1999). Formulas relating $\mathbb{K} \mathbb{L}$ stability estimates of discretetime and sampled-data nonlinear systems. Systems \& Control Letters, vol. 38, p. 49-60.

Souza M., Deaecto G., Geromel J., Daafouz J. (2012, July). Self-triggered linear quadratic networked control. In Control Automation (MED), 2012 20th Mediterranean Conference on, p. 948-953. 